\title{
PERFIL DIFERENCIAL DE TRASTORNOS DE PERSONALIDAD EN EL CONSUMO DE DROGAS Y MALTRATO
}

\section{PERSONALITY DISORDER PROFILES IN DRUG ABUSE AND ABUSE}

\author{
Eva Castillo FernándeZ ${ }^{1}$, EnRiQue J. Gómez SÁnCHEZ ${ }^{1}$, \\ JOSÉ LuÍS MATA-MARTÍN ${ }^{2}$, ISABEL RAMÍREZ UCLES ${ }^{3}$ Y \\ FRANCISCA LÓPEZ TORRECILLAS ${ }^{2}$
}

Cómo referenciar este artículo/How to reference this article:

Castillo Fernández, E., Gómez Sánchez, E. J., Mata-Martín, J. L., Ramírez Uclés, I. y López Torrecillas, F. (2016). Perfil diferencial de trastornos de personalidad en el consumo de drogas y maltrato [Personality disorder profeiles in drug abuse and abuse]. Acción Psicológica, 13(1), 31-40. http://dx.doi.org/10.5944/ap.13.1.17394

\section{Resumen}

Existe evidencia empírica que demuestra la relación entre patrones de personalidad, tipos de delitos violentos y consumo de drogas o alcohol. El presente estudio tiene como objetivo examinar la tasa de prevalencia de trastornos de personalidad en prisión (consumidores de drogas y maltratadores), utilizando como grupo control población encarcelada sin este tipo de delitos.
\end{abstract}

Participaron en este estudio 45 hombres del Centro Penitenciario de Albolote, Granada (15 consumidores de drogas, 15 maltratadores y 15 controles) que fueron seleccionados mediante la Escala de Severidad de la Adicción (EUROPASI; Bobes et al., 1996) y la Escala de Tácticas de Conflicto (CTS2; Strauss et al., 1996). Para evaluar los trastornos de personalidad se utilizó el Inventario Clínico Multiaxial de Millon. Encontramos que el grupo de consumo de drogas puntúa más alto en los trastornos de personalidad histriónico, antisocial, límite y agresivo-sádico en comparación con el grupo de maltrato y control y que el grupo de maltrato puntúa más

Correspondencia: Francisca López Torrecillas, Departamento de Personalidad, Evaluación y Tratamiento Psicológico Facultad de Psicología, Campus de Cartuja s/n, Universidad de Granada

Email: fcalopez@ugr.es

${ }^{1}$ Centro Penitenciario Albolote, España.

${ }^{2}$ Centro de Investigación Mente, Cerebro y Comportamiento (CIMCYC), España.

${ }^{3}$ Departamento de Psicología Básica II. Facultad de Psicología, UNED, España.

Recibido: 20 de ocrubre de 2016.

Aceptado: 20 de diciembre de 2016. 
alto en el trastorno de personalidad dependiente en comparación con el grupo de drogas y control. Nuestros resultados resaltan la necesidad de tener en cuenta la existencia de trastornos de personalidad en población clínica con el fin de diseñar estrategias de intervención y prevención para el consumo de drogas y el maltrato.

Palabras clave: Trastornos de personalidad; consumo de drogas; maltrato.

\begin{abstract}
There is empirical evidence showing the relationship between personality disorders, violence crime and substance abuse problem. This study aims to examine the prevalence of personality disorders in prison populations (drug abusers and abusers), using as a control group a population imprisoned without these types of crimes. The participants were 45 men within the prison in Albolote, Granada (15 drug abusers, 15 abusers and 15 control) and were selected following the Severity Addiction Scale (EUROPASI; Bobes et al., 1996) and the Conflict Tactics Scale (CTS2; Strauss et al., 1996). The Millon Clinical Multiaxial Inventory was used to assess personality disorders. We found that drug abusers had higher scores than abusers and controls in histrionic, antisocial, borderline and aggressive-sadistic, and abusers had higher scores than drug abusers and controls in dependent personality disorder. Our results highlight the need to take into account the existence of personality disorders in clinical population in order to design prevention and intervention strategies for drug abuse and domestic violence.
\end{abstract}

Keywords: Personality disorders; drug abuse; abusers.

\section{Introducción}

En la actualidad, el consumo de drogas y el maltrato constituyen dos de los problemas sociales más graves, complejos y difíciles de abordar desde todas las perspec- tivas, incluida la científica. Existen numerosos datos epidemiológicos que vinculan estrechamente maltrato y consumo de drogas (Arteaga, Fernández-Montalvo y López-Goñi, 2012). El consumo de drogas reduce los controles inhibitorios de la violencia, al tiempo que disminuye el miedo ante situaciones de riesgo y los sentimientos de culpa que normalmente se producirían en ausencia de dicho consumo. Además, ciertas conductas consideradas ilegales (por ejemplo, conducir de manera temeraria) facilitan o instan al consumo de drogas, y recíprocamente, el consumo de drogas o sus efectos y consecuencias, pueden acabar instando a la ejecución de delitos (por ejemplo, el maltrato físico o psicológico). Aunque el consumo de drogas y alcohol, por si solos, serían insuficientes para explicar la conducta violenta en sí misma, existe evidencia empírica que demuestra la relación entre patrones de personalidad, tipos de delitos violentos y consumo de drogas o alcohol (Cunha y Gonçalves, 2013; DeLisi, Vaughn, Salas-Wright y Jennings, 2015).

El consumo de drogas antes de entrar en prisión también es una realidad palpable en una elevada proporción de internos. Paralelamente, la presencia de trastornos de la personalidad en personas con problemas de consumo de drogas se ha convertido en un tema de interés, no sólo por la alta prevalencia de estos problemas sino también por la necesidad de tenerlos en cuenta en el momento de organizar y estructurar la intervención. En este sentido Casares-López et al. (2010) sostienen que la comorbilidad entre el consumo de drogas y los trastornos de personalidad es mayor cuando el contexto de estudio se circunscribe a la prisión, donde los drogodependientes en régimen penitenciario presentan más trastornos de personalidad y más graves que los drogodependientes que están en tratamiento en régimen extrapenitenciario. Los estudios (Edens, Kelley, Lilienfeld, Skeem y Douglas, 2015; Mahmood, Tripodi, Vaughn, Bender y Schwartz, 2012; Marco et al., 2015) concluyen que los trastornos de personalidad de los drogodependientes en régimen penitenciario son en primer lugar los trastornos que pertenecen al cluster $\mathrm{B}$, concretamente el límite y el antisocial; en segundo lugar, los del cluster $\mathrm{C}$, trastorno por evitación, pasivo-agresivo y obsesivo compulsivo; y en tercer lugar, el cluster A, destacando el esquizoide. 
Por otro lado, en los últimos años se ha producido un aumento considerable de las investigaciones relacionadas con los hombres que agreden física y/o psicológicamente a la mujer. Ello ha traído consigo un conocimiento más preciso de los trastornos de personalidad en delincuentes con antecedentes de delitos sexuales en comparación con otro tipo de delitos. Estudios recientes (Arteaga et al., 2012; Schroeder, Iffland, Hill, Berner y Briken, 2013; Vaughn et al., 2011) ponen de manifiesto un predominio de los trastornos de depresión mayor, de personalidad paranoide, obsesivo-compulsivo y esquizoide en delincuentes con antecedentes de delitos sexuales. Finalmente resaltar el estudio de Fernández-Montalvo y Echeburúa (2008) que analiza los trastornos de personalidad y psicopatía asociados a una muestra de maltratadores contra la mujer ingresados en prisión. Los autores encontraron que el $34.2 \%$ de la muestra de agresores presentaba un trastorno dependiente de la personalidad. En la misma línea, Loinaz, Ortiz-Tallo, Sánchez y Ferragut (2011) constataron, de nuevo, la existencia de los dos tipos de agresores contra la pareja. El tipo 1 considerado como normalizado se caracterizó por presentar los trastornos de personalidad narcisista, histriónico y obsesivo-compulsivo y el tipo 2 considerado como antisocial que presentaba los trastornos de personalidad antisocial, agresivo-sádico, pasivo-agresivo y paranoide y trastornos de ansiedad, dependencia de sustancias y de alcohol, delirante y bipolar.

Sin embargo, las investigaciones llevadas a cabo hasta la fecha muestran una gran heterogeneidad debido, entre otras causas, a la falta de control sobre si los agresores consumen o no consumen drogas.

El presente estudio tiene como objetivo analizar en población clínica - consumidores de droga y maltratadores- la tasa de prevalencia de trastornos de personalidad medidos a través el Inventario Clínico Multiaxial de Millon (MCMI-II) (1999). Nuestra hipótesis de partida es, por tanto, que existen diferentes trastornos de personalidad en consumidores de drogas y maltratadores, ambos encarcelados por dicho motivo.

\section{Método}

\section{Participantes}

Participaron en este estudio 45 hombres encarcelados en el Centro Penitenciario de Albolote de Granada (15 con historia de consumo de drogas, 15 con historia de maltrato y 15 personas sin historia de consumo de drogas ni maltrato también internos). Los participantes fueron seleccionados de acuerdo con los criterios del Personal Técnico Especializado de la prisión, de su historial delictivo y de escalas específicas para evaluar la gravedad de la adicción (Escala de Severidad de la Adicción (EUROPASI; Bobes, Gónzález, Sáiz y Bousoño, 1996) y el maltrato (Escala de Tácticas de Conflicto (CTS2; Strauss, Hamby, Boney-McCoy y Sugarman, 1996). Los criterios de inclusión fueron estar cumpliendo condena en el Centro Penitenciario de Albolote y participar voluntariamente en el estudio, después haber firmado la hoja de consentimiento informado. Además, en el caso del grupo de drogodependencias los criterios fueron tener un problema de adicción a las drogas y/o al alcohol y no cumplir o haber cumplido condena por un delito de maltrato a la pareja. En el caso del grupo de maltrato los criterios fueron estar condenado por un delito de maltrato a la pareja y no tener un problema de adicción a las drogas y/o al alcohol, y finalmente en el caso del grupo control los criterios fueron no tener un problema de adicción a las drogas y/o al alcohol y no cumplir o haber cumplido condena por un delito de maltrato a la pareja. Los criterios de exclusión fueron tener una edad superior a 50 años, enfermedad física o psiquiátrica (esquizofrenia y/o depresión) y estar bajo tratamiento psicofarmacológico. Se excluyo un caso por invalidación en las respuestas del Inventario Clínico Multiaxial de Millon (MCMI-II) de Millon (1999) por lo que los análisis estadísticos se realizaron con 44 participantes.

Los grupos quedaron igualados en edad, nivel de estudios, profesión (ver Tabla 1). 
Tabla 1

Variables sociodemográficas

\begin{tabular}{|c|c|c|c|c|}
\hline & & Grupos & & $F / X^{2}$ \\
\hline Edad (media y SD) & $35.64(5.35)$ & $37.53(9.04)$ & $36.66(9.80)$ & 0.831 \\
\hline Años de escolarización (N) & & & & 0.64 \\
\hline 6 & 35.72 & 60 & 0 & \\
\hline 7 & 7.14 & 0 & 0 & \\
\hline 8 & 42.86 & 26.67 & 66.66 & \\
\hline 10 & 0 & 30 & 6.67 & \\
\hline 11 & 0 & 13.33 & 13.33 & \\
\hline 12 & 7.14 & 4 & 0 & \\
\hline 13 & 7.14 & 0 & 0 & \\
\hline 15 & 0 & 0 & 6.67 & \\
\hline 17 & 0 & 0 & 6.67 & \\
\hline Profesión & & & & 0.348 \\
\hline Sin formación previa & 92.86 & 86.66 & 66.67 & \\
\hline Con formación previa & 7.14 & 6.67 & 13.33 & \\
\hline Empresario & 0 & 6.67 & 20 & \\
\hline Estado civil & & & & 0.008 \\
\hline Casado & 0 & 0 & 26.67 & \\
\hline Viudo & 0 & 0 & 6.66 & \\
\hline Separado & 21.43 & 26.67 & 6.66 & \\
\hline Divorciado & 7.14 & 33.33 & 20 & \\
\hline Soltero & 64.29 & 1333 & 13.34 & \\
\hline Convivencia en pareja & 7.14 & 26.67 & 26.67 & \\
\hline
\end{tabular}

\section{Instrumentos}

Escala de Severidad de la Adicción (EUROPASI; Bobes et al., 1996). Versión europea del instrumento Addiction Severity Index (ASI; McLellan, Luborsky, O'Brein y Woody, 1980). Es una entrevista estructurada de unos cuarenta minutos de duración que abarca ocho aspectos de la problemática del dependiente, tales como problemas médicos, laborales, uso de alcohol y drogas, legales, familiares, psicológicos y otros problemas. Se efectúa también una valoración de la actitud y motivación del adicto. Una vez finalizada la entrevista el evaluador da una puntuación sobre la gravedad estimada en cada una de las áreas evaluadas. Para ello se puede emplear el Índice de Gravedad del Entrevistador (IGE; López-Goñi, Fernández-Montalvo y Arteaga, 2012) con un rango de 0-9, indicando que a mayor puntuación, mayor gravedad de la adicción. Es muy utilizada tanto desde la perspectiva clínica como desde la institucional (González-Saiz et al., 2002). La versión española empleada en este estudio es de Bobes et al. (1996).

Escala de Tácticas de Conflicto (CTS-2; Strauss et al., 1996). Contempla la violencia como una táctica errónea de resolución de conflictos. Mide acontecimientos, procesos o intentos de resolución de conflictos y las tácticas utilizadas para ello. Consta de 39 ítems duplicados, es decir, 39 preguntas para cada miembro de la pareja (78 ítems en total). El tiempo medio de aplicación no debe superar los 15 minutos. El tiempo de referencia en la subescala de prevalencia es de un año, aunque en algunos casos puede limitarse a seis meses, especialmente cuando se trata de medir la respuesta a tratamientos terapéuticos. Los ítems están representados 
en varias subescalas: Negociación, Agresión Psicológica, Agresión Física, Coerción Sexual y Daño o lesiones. Ha sido adaptada y validada a la población española por Graña, Andreu, Peña y Rodríguez-Biezma (2013).

Inventario Clínico Multiaxial de Millon (MCMI-II; Millon, 1999). Se trata de un cuestionario autoinformado de 175 ítems con alternativa de respuesta dicotómica, de tipo verdadero-falso. Este test permite la evaluación de 13 trastornos de personalidad y de nueve síndromes clínicos diferentes. Dentro de las escalas de personalidad se distinguen 10 estilos básicos y tres escalas de personalidad patológica. En cuanto a las escalas de síndromes clínicos se obtienen seis síndromes clínicos de gravedad moderada y tres síndromes clínicos de gravedad extrema. Incorpora también cuatro escalas de control para determinar posibles sesgos a la hora de contestar a las preguntas validez, sinceridad, deseabilidad y alteración. Las escalas clínicas son básicas (esquizoide, fóbica, dependiente, histriónica, narcisista, antisocial, agresiva-sádica, compulsiva, pasivo-agresiva y autodestructiva), patológica (esquizotípica, límite y paranoide), síndromes clínicos de gravedad moderada (ansiedad, histeriforme, hipomanía, neurosis depresiva, abuso de alcohol y abuso de drogas) y síndromes clínicos de gravedad extrema: pensamiento psicótico, depresión mayor y trastorno delirante. En este estudio se utiliza la adaptación española de TEA.

\section{Procedimiento}

Para poder llevar a cabo esta investigación se solicitó la autorización necesaria al Centro Penitenciario de Albolote perteneciente a la Dirección General de Instituciones Penitenciarias dependiente del Ministerio de Interior. Los 45 participantes fueron seleccionados previamente y entrevistados individualmente para confirmar los criterios de inclusión y se les ofreció participar voluntariamente en la investigación. Los 45 participantes que cumplían con los criterios de inclusión fueron debidamente informados sobre los objetivos del estudio, la metodología empleada y manifestaron su consentimiento por escrito. Posteriormente completaron las escalas e inventarios descritos en el apartado de instrumentos. A los participantes se les recordaba, al inicio de la sesión, su derecho a interrumpir el procedimiento en cualquier momento. Al finalizar la sesión recibirán una breve explicación de los objetivos del estudio y se les agradece su participación.

\section{Resultados}

Para comprobar la hipótesis, se realizó un Análisis Multivariado de la Varianza (MANOVA), para un diseño factorial, utilizando las variables grupo (drogodependencias, maltrato y control) como variable independiente y las variables derivadas del Inventario Clínico Multiaxial de Millon (MCMI-II) como dependientes (esquizoide, fóbica, dependiente, histriónica, narcisista, antisocial, agresiva-sádica, compulsiva, pasivo-agresiva, autodestructiva, esquizotípica, límite, paranoide, ansiedad, histeriforme, hipomanía, neurosis depresiva, abuso de alcohol, abuso de drogas, pensamiento psicótico, depresión mayor y trastorno delirante).

Los resultados indicaron que había diferencias estadísticamente significativas entre los grupos (Wilks' Lambda $=0,034, \mathrm{~F}_{44}, 40=4.005, p=.000 ; \eta=0.82$ ). Puesto que el MANOVA mostró resultados estadísticamente significativos, se procedió a realizar ANOVAs para cada variable dependiente. Dichos ANOVAs mostraron que había diferencias estadísticamente significativas en el trastorno de personalidad dependiente $\left(\mathrm{F}_{2,41}=5.94\right.$; $\mathrm{Mce}=3501.056$, $p=.005)$, histriónica $\left(\mathrm{F}_{2}, 41=3.92, \quad\right.$ Mce $=2343.159$, $p=.028)$, antisocial $\left(\mathrm{F}_{2,41}=13.74, \quad\right.$ Mce $=8092.474$, $p=.000), \quad$ agresiva-sádica $\quad(\mathrm{F} 2, \quad 41=9.88$, Mce $=5514.759, \quad p=.000), \quad$ límite $\left(F_{2}, 41=7.93\right.$, Mce $=2970.744, \quad p=.001)$, abuso de alcohol $\left(\mathrm{F}_{2}\right.$, ${ }_{41}=31.92$, Mce $\left.=13142.752, p=.000\right)$, abuso de drogas $\left(\mathrm{F}_{2,41}=35.14\right.$, Mce $\left.=13719.594, p=.000\right)$ y depresión mayor $\left(\mathrm{F}_{2,41}=4.41\right.$, Mce $\left.=2072.906, p=.018\right)$. Aunque no encontramos diferencias estadísticamente significativas resaltamos que las variables esquizoide $\left(\mathrm{F}_{2,41}=2.78, \quad \mathrm{Mce}=2089.752, \quad p=.074\right), \quad$ fóbica $\left.\mathrm{F}_{2,41}=2.88, \quad \mathrm{Mce}=1958.973, \quad p=.068\right), \quad$ compulsiva $\left(\mathrm{F}_{2,41}=3.11, \mathrm{Mce}=1229.648, p=.055\right)$, pasivo-agresiva $\left(\mathrm{F}_{2,41}=3.10, \quad \mathrm{Mce}=1987.217, \quad p=.056\right)$, pensamiento psicótico $\left(\mathrm{F}_{2,41}=2.97, \quad\right.$ Mce $\left.=2453.298, \quad p=.062\right)$ se 
acercan a la significatividad, por lo que podríamos pensar en las diferencias clínicas de dichas variables.

Los resultados de las pruebas de contraste "post hoc" de comparaciones múltiples entre los tres grupos (Tukey) pueden verse en la Tabla 2, donde se presentan los datos de los trastornos de personalidad dependiente, histriónica, antisocial, agresiva-sádica, límite, abuso de alcohol, abuso de drogas y depresión mayor.

Como puede verse en la Tabla 2, el grupo de maltrato presenta puntuaciones significativamente más altas en trastorno de personalidad dependiente que el grupo de drogodependencias; también el grupo de maltrato presenta puntuaciones significativamente más altas que el grupo control. Respecto al trastorno de personalidad histriónico, las puntuaciones son más altas en el grupo de drogodependencias que en el grupo control. En el trastorno de personalidad antisocial encontramos diferencias entre el grupo de drogodependencias y los grupos de maltrato y control siendo las puntuaciones más altas en el grupo de drogodependencias. Igualmente, el grupo de drogodependencias puntúa más alto en el trastorno de personalidad agresiva-sádica que el grupo de maltrato y que el grupo control. Con respecto al trastorno de personalidad compulsivo, es el grupo de control el que presenta las puntuaciones más altas en comparación con el grupo de drogodependencias y en el caso del trastorno de personalidad pasivo-agresiva las diferencias son inversas entre el grupo de drogodependencias y el grupo control, este último puntúa más bajo. En cuanto al trastorno limite de la personalidad, las puntuaciones son mas altas en el grupo de drogodependencias que en el de maltrato y que en el grupo control. En cuanto a los síndromes clínicos

Tabla 2

Media, desviación típica y significatividad de los grupos en los Trastornos de Personalidad

\begin{tabular}{|c|c|c|c|c|c|}
\hline Trastornos de Personalidad & $\begin{array}{l}\text { DROGAS } \\
\text { Media (DT) }\end{array}$ & $\begin{array}{l}\text { MALTRATO } \\
\text { Media (DT) }\end{array}$ & $\begin{array}{l}\text { CONTROL } \\
\text { Media (DT) }\end{array}$ & $\boldsymbol{F}$ & p-Tukey \\
\hline Esquizoide & $67.21(26.74)$ & $44.93(30.09)$ & $48.00(25.15)$ & 2.78 & \\
\hline Fóbico & $53.21(22.17)$ & $37.53(27.27)$ & $30.40(28.16)$ & 2.88 & \\
\hline Dependiente & $60.14(26.46)$ & $88.60(19.68)$ & $64.13(26.21)$ & $5.94^{* *}$ & $1<2^{* *}>3^{*}$ \\
\hline Histriónico & $78.71(28.86)$ & $75.20(20.89)$ & $55.33(23.23)$ & $3.92^{*}$ & $1>3^{*}$ \\
\hline Narcisista & $70.28(28.28)$ & $67.67(31.03)$ & $52.53(33.44)$ & 1.40 & \\
\hline Antisocial & $84.14(18.38)$ & $42.80(24.74)$ & 43.13(28.27) & $13.74^{* * *}$ & $1>2^{* * *} ; 1>3^{* *}$ \\
\hline Agresivo-sádico & $68.14(26.85)$ & $30.60(18.09)$ & $39.60(25.24)$ & $9.88^{* * *}$ & $1>2^{* * *} ; 1>3^{* *}$ \\
\hline Obsesivo-compulsivo & $84.71(26.36)$ & $96.13(17.78)$ & $103.00(13.95)$ & $3.11^{*}$ & $1<3^{*}$ \\
\hline Pasivo-agresivo & $47.50(23.58)$ & $24.33(21.11)$ & $32.53(30.29)$ & $3.10^{*}$ & $1>2^{*}$ \\
\hline Autodestructivo & $52.07(18.97)$ & $43.60(29.02)$ & $35.00(27.36)$ & 1.61 & \\
\hline Esquizotípico & $70.36(23.89)$ & $55.00(28.54)$ & $51.20(29.79)$ & 1.94 & \\
\hline Límite & $61.43(17.48)$ & $39.73(18.41)$ & $34.20(21.77)$ & $7.93^{* *}$ & $1>2^{*} ; 1>3^{* *}$ \\
\hline Paranoide & $80.00(28.06)$ & $66.47(33.85)$ & $64.13(37.03)$ & 0.95 & \\
\hline Ansiedad & $48.28(21.70)$ & $45.47(23.99)$ & $37.93(8.78)$ & 1.13 & \\
\hline Histeriforme & $53.07(28.29)$ & $52.00(20.90)$ & $39.93(13.90)$ & 1.68 & \\
\hline Hipomanía & $71.14(31.56)$ & $57.60(26.40)$ & $49.67(25.31)$ & 2.20 & \\
\hline Neurosis-depresivo & $51.21(18.95)$ & $47.07(25.60)$ & $37.07(11.11)$ & 2.04 & \\
\hline Abuso alcohol & $83.93(18.42)$ & $35.20(25.13)$ & $28.40(16.11)$ & $31.92^{\star * *}$ & $1>2^{* * *} ; 1>3^{* * *}$ \\
\hline Abuso drogas & $89.78(11.05)$ & $38.40(22.33)$ & $34.20(23.05)$ & $35.14^{\star * *}$ & $1>2^{* * *} ; 1>3^{* * *}$ \\
\hline $\begin{array}{l}\text { Pensamiento psicótico } \\
\text { Depresión mayor }\end{array}$ & $\begin{array}{l}66.78(21.95) \\
41.14(25.51)\end{array}$ & $\begin{array}{l}44.67(31.24) \\
31.40(23.68)\end{array}$ & $\begin{array}{l}43.60(31.52) \\
17.40(14.51)\end{array}$ & $\begin{array}{l}2.97 \\
4.41^{*}\end{array}$ & $1>3^{*}$ \\
\hline Delirio psicótico & $80.36(28.63)$ & $77.67(32.98)$ & $72.73(34.28)$ & 0.21 & \\
\hline
\end{tabular}

Nota. ${ }^{\star \star \star} p<.001,{ }^{* \star} p<.01, p<.05$ 
resaltamos que el grupo de drogas puntúa más alto tanto en el abuso de alcohol como en el abuso de drogas que el grupo de maltrato y de control. También el grupo de drogas puntúa más alto en depresión mayor que el grupo control.

\section{Discusión y Conclusiones}

El presente estudio contribuye a conocer, con más detalle, la presencia de posibles alteraciones de personalidad en una muestra de hombres ingresados en el Centro Penitenciario de Albolote; esto es, permite clarificar el solapamiento existente entre las conductas generadas por el consumo de drogas y trastornos de personalidad y aquellas generadas por los sujetos que no consumen drogas pero que se caracterizan por cometer actos criminales, engaños y falta de responsabilidad, egocentrismo, exageración en la expresión de emociones, falta de emotividad real y cambios rápidos de estado emocional que pueden ser, tanto la expresión de un trastorno de personalidad como las consecuencias del consumo de drogas y/o alcohol. Del mismo modo, nuestro estudio nos permite comparar el perfil psicopatológico de los grupos analizados.

Por lo que se refiere a las características sociodemográficas de nuestra muestra encontramos diferencias estadísticamente significativas en la variable estado civil, indicando que el grupo de drogodependientes se encuentra mayoritariamente soltero. Estos resultados son consonantes con los obtenidos por Casares-López et al. (2010), donde tanto el perfil del usuario que ingresaba en comunidad terapéutica, como el perfil del usuario que ingresaba en prisión se correspondían mayoritariamente con varones solteros. Por otro lado, encontramos que el grupo de maltrato presenta un mayor porcentaje de divorciados en comparación con el grupo de drogodependencias y el grupo control, resultado que también es consonante con el obtenido por Fernández-Montalvo y Echeburúa (2008), que observan un predominio de personas divorciadas $(59.2 \%)$, muy por encima de los sujetos solteros $(23.7 \%)$, casados $(13.1 \%)$ y viudos $(3.9 \%)$.
Resulta llamativo que, en nuestra muestra de estudio, el grupo de drogodependencias y de maltrato no estén casados en comparación con el grupo control. Estos resultados son evidentes, dado que, una de las características del grupo de consumidores de drogas es la carencia de relaciones sociales y en el caso del grupo de maltrato, este resultado, podría deberse a las consecuencias derivadas de la naturaleza del delito. Estos resultados son parecidos a los encontrados en los estudios de Casares-López et al. (2010) quienes encuentran que solo un $13.9 \%$ de la muestra de drogodependientes y un $13.1 \%$ de la muestra de maltratadores respectivamente estaban casados. Destacar también que encontramos un mayor porcentaje de separados en el grupo de drogodependencias y en el grupo de maltrato en comparación con el grupo control. Por último, señalar como un dato significativo que en el grupo de drogodependencias solo un $7.14 \%$ convivían en pareja frente a un $26.67 \%$ que representa a los grupos de maltrato y control. Estos resultados reflejan el bajo nivel de adaptación y la falta de estrategias de afrontamiento que caracterizan a los consumidores de drogas y/o alcohol.

En relación a los trastornos de personalidad, los resultados obtenidos muestran diferencias estadísticamente significativas en cinco de los trece trastornos de personalidad evaluados (dependiente, histriónico, antisocial, agresivo-sádico y límite), en dos síndromes clínicos (abuso de alcohol y abuso de drogas) y en un síndrome grave (depresión mayor); y diferencias que se acercan a la significatividad en el trastorno compulsivo de la personalidad y en el trastorno pasivo/agresivo de la personalidad. Estos resultados son congruentes con los obtenidos por Edens et al. (2015), Mahmood et al. (2012) y Marco et al. (2015) que también encontraron diferencias estadísticamente significativas, en una muestra de personas condenadas por delitos violentos, en los trastornos de la personalidad dependiente, compulsivo, antisocial, límite, abuso de alcohol, abuso de drogas y depresión mayor.

Respecto a los tipos de trastornos de personalidad encontrados y a las diferencias entre los grupos, 
nuestros resultados muestran que el grupo de maltrato presenta puntuaciones significativamente más altas en trastorno de personalidad dependiente que los grupos de drogodependencias y control. Este resultado es consistente con los encontrados por FernándezMontalvo y Echeburúa (2008) en el cual manifiestan que el $34.2 \%$ de los casos de su muestra de agresores presentaban un trastorno dependiente de la personalidad. En cuanto al trastorno de personalidad histriónico, las puntuaciones son más altas en el grupo de drogodependencias que en el grupo control. Este resultado es coherente con el de otros estudios (Edens et al., 2015; Mahmood et al., 2012; Marco et al., 2015). En el trastorno de personalidad antisocial encontramos diferencias entre el grupo de drogodependencias y los grupos de maltrato y control, siendo las puntuaciones más altas en el grupo de drogodependencias. Nuestros resultados van en la misma línea que los obtenidos por otros autores (Edens et al., 2015; Mahmood et al., 2012; Marco et al., 2015).

Así, el grupo de drogodependencias puntúa más alto en el trastorno de personalidad agresiva-sádica que el grupo de maltrato y que el grupo control. Este resultado es similar al del estudio de Loinaz et al. (2011) que obtienen que el tipo 2, denominado antisocial y con dependencia de sustancias y alcohol, destaca por puntuaciones significativamente superiores en la escala de personalidad agresivo-sádica. Con respecto al trastorno de personalidad obsesivo-compulsivo el grupo control presenta las puntuaciones más altas que el grupo de drogodependencias, este resultado es concordante con el obtenido por Ortiz-Tallo et al. (2006) en el que los grupos no dependientes de drogas o alcohol puntuaban más alto en este trastorno. En relación al trastorno pasivo/agresivo es el grupo de drogodependencias el que presenta las puntuaciones más altas que el grupo control, este resultado va en la misma línea que el obtenido por López-Durán y Becoña (2006) quienes concluyen que los consumidores de drogas presentan mayores porcentajes de prevalencia de trastornos de personalidad agresivos.

Nuestros resultados también ponen de manifiesto que el grupo de drogodependencias presenta puntuaciones más altas en el trastorno límite de la personalidad que los grupos de maltrato y control. Estos resultados son consonantes con los obtenidos en estudios previos (Edens et al., 2015; Mahmood et al., 2012; Marco et al., 2015).

Por otro lado, en lo que a los síndromes clínicos se refiere, resaltamos que el grupo de drogas puntúa más alto tanto en el abuso de alcohol como en el abuso de drogas que el grupo de maltrato y de control, resultado que concuerda con el obtenido por Casares-López et al. (2010), los cuales encentran que el grupo de drogodependientes en prisión presentan puntuaciones más altas en la escala de abuso de alcohol y drogas que el grupo de drogodependientes ingresado en comunidad terapéutica. Finalmente, el grupo de drogas puntúa más alto en depresión mayor que el grupo control, datos que están en sintonía con los obtenidos por Vaughn et al. (2011) donde los hombres no consumidores eran significativamente menos propensos a ser diagnosticados de un trastorno de depresión mayor.

En general, constatamos la elevada prevalencia de los trastornos de personalidad entre los drogodependientes, por lo que podemos concluir que, tal y como señalaron Casares-López et al. (2010), el grupo de internos penados con consumo de drogas presentan más trastornos de la personalidad que el resto de grupos. De manera similar, también constatamos, de acuerdo con Edens et al. (2015), Mahmood et al. (2012) y Marco et al. (2015), que tres de los cuatros trastornos más significativos en los drogodependientes se encuentran incluidos en la categoría B (histriónico, antisocial y limite).

Un aspecto importante a señalar en este estudio es que el grupo de drogodependientes eran pacientes en tratamiento que llevaban más de seis meses abstinentes, además de no tener tratamiento psicofarmacológico, con lo que quedaría minimizada la posibilidad que sintomatología asociada al consumo de drogas pueda ser confundida con síntomas de los trastornos de personalidad. 
Por tanto, nuestros resultados indican la existencia de trastornos de personalidad en población penitenciaria y la necesidad de seguir investigando en esta línea con el fin de diseñar estrategias de prevención para el consumo de drogas y maltrato.

\section{Referencias}

Arteaga A., Fernández-Montalvo J. y López-Goñi J. J. (2012). Diferencias en variables de personalidad en sujetos adictos a drogas con $y$ sin conductas violentas contra la pareja. [Differences in personality variables in drug-addicted patients with and without intimate partner violence]. Acción Psicológica, 9(1),

19-32. http://dx.doi.org/10.5944/ap.9.1.435.

Bobes, J., González, M. P., Sáiz, P. A. y Bousoño, M. (1996). Índice Europeo de severidad de la adicción: EuropASI. Versión española. En Libro de Actas IV Reunión Interregional de Psiquiatría (pp.201-218). Gijón, España: Bobes- García.

Casares-López, M. J., González-Menéndez, A., TorresLobo, M., Secades-Villa, R., Fernández-Hermida, J. R. y Álvarez, M. (2010). Comparación del perfil psicopatológico y adictivo de dos muestras de adictos en tratamiento: En prisión y en comunidad terapéutica. International Journal of Clinical and Health Psychology, 10(2), 225-243. Recuperado de http://search.proquest.com/docview/742981227?acc ountid=14542.

Cunha, O. y Gonçalves, R. A. (2013). Intimate partner violence offenders: Generating a data-based typology of batterers and implications for treatment/La violencia de pareja: Generación de una tipología de maltratadores basada en los datos e implicaciones para el tratamiento. The European Journal of Psychology Applied to Legal Context, 5(2), 131-139. Recuperado de http://search.proquest.com/docview/1404742575?ac countid=14542.
DeLisi, M., Vaughn, M. G., Salas-Wright, C. y Jennings, W. G. (2015). Drugged and dangerous: Prevalence and variants of substance use comorbidity among seriously violent offenders in the united states. Journal of Drug Issues, 45(3), 232-248. http://dx.doi.org/10.1177/0022042615579237.

Edens, J. F., Kelley, S. E., Lilienfeld, S. O., Skeem, J. L. y Douglas, K. S. (2015). DSM-5 antisocial personality disorder: Predictive validity in a prison sample. Law and Human Behavior, 39(2), 123-129. http://dx.doi.org/10.1037/lhb0000105.

Fernández-Montalvo, J. y Echeburúa, E. (2008). Trastornos de personalidad y psicopatía en hombres condenados por violencia grave contra la pareja. Psicothema, 20(2), 193-198. Recuperado de http://search.proquest.com/docview/621937936?acc ountid $=14542$.

González-Saiz, F., Salvador, J. M., Martínez-Delgado, J. M., López-Cárdenas, A., Ruz, I. y Guerra, D. (2002). El Addiction Severity Index (ASI): a propósito de una revisión [The Addiction Severity Index (ASI): Report of a Review]. En I. Iraurgi y F. González-Saiz (Eds.), Instrumentos de evaluación en drogodependencias (pp. 235-265). Madrid, España: Aula Médica.

Graña, J. L., Andreu, J. M., Peña, M. E. y RodríguezBiezma, M. J. (2013). Validez factorial y fiabilidad de la "escala de tácticas para el conflicto revisada" (revised conflict tactics scale, CTS2) en población adulta española. Psicología Conductual, 21(3), 525543. Recuperado de http://search.proquest.com/docview/1476261014?ac countid $=14542$.

Loinaz, I., Ortiz-Tallo, M., Sánchez, L. M. y Ferragut, M. (2011). Clasificación multiaxial de agresores de pareja en centros penitenciarios. International Journal of Clinical and Health Psychology, 11(2), 249-268. Recuperado de http://search.proquest.com/docview/897336549?acc ountid=14542. 
López- Durán, A. L. y Becoña, E (2006). Patrones y trastornos de personalidad en personas con dependencia de la cocaína en tratamiento. Psicothema, 18(3), 578-583. Recuperado de http://search.proquest.com/docview/621503952?acc ountid $=14542$.

López-Goñi, J., Fernandez-Montalvo, J. y Arteaga, A. (2012). Predictive validity of the EuropASI: Clinical diagnosis or composite scoring? Journal of Substance Abuse Treatment, 42(4), 392-399. http://dx.doi.org/10.1016/j.jsat.2011.09.011.

Mahmood, S. T., Tripodi, S. J., Vaughn, M. G., Bender, K. A. y Schwartz, R. D. (2012). Effects of personality disorder and impulsivity on emotional adaptations in prison among women offenders. Psychiatric Quarterly, 83(4), 467-80. http://dx.doi.org/10.1007/s11126-012-9215-5.

McLellan, A. T., Luborsky, L., Woody, G. E. y O'Brien, C. P. (1980). An improved diagnostic evaluation instrument for substance abuse patients: The addiction severity index. Journal of Nervous and Mental Disease, 168(1), 26-33. http://dx.doi.org/10.1097/00005053-19800100000006 .

Marco, A., Antón, J. J., Saiz de, 1. H., de Juan, J., Faraco, I., Caylà, J. A., ... Group, P. (2015). Personality disorders among spanish prisoners starting hepatitis C treatment: Prevalence and associated factors. Psychiatry Research, 230(3), 749-756. http://dx.doi.org/10.1016/j.psychres.2015.11.016.

Millon, T. (1999). MCMI-II. Inventario clínico multiaxial de Millon-II. Manual. Adaptación española (Adaptación española de A. Ávila y F. Jiménez). Madrid, España: TEA.

Ortiz-Tallo, M., Fierro, A., Blanca, M. J., Cardenal, V. y Sánchez, L. M. (2006). Factores de personalidad y delitos violentos. Psicothema, 18(3), 459-464. Recuperado http://search.proquest.com/docview/621455153?acc ountid $=14542$.
Schroeder, M., Iffland, J. S., Hill, A., Berner, W. y Briken, P. (2013). Personality disorders in men with sexual and violent criminal offense histories. Journal of Personality Disorders, 27(4), 519-30. http://dx.doi.org/101521 pedi201226050.

Straus, M. A., Hamby, S. L., Boney-McCoy, S. y Sugarman, D. B. (1996). The revised conflict tactics scales (CTS2): Development and preliminary psychometric data. Journal of Family Issues, 17(3), 283-316. Recuperado de http://search.proquest.com/docview/61541186?acco untid $=14542$.

Vaughn, M. G., Fu, Q., Wernet, S. J., DeLisi, M., Beaver, K. M., Perron, B. E. y Howard, M. O. (2011). Characteristics of abstainers from substance use and antisocial behavior in the united states. Journal of Criminal Justice, 39(3), 212. Recuperado de http://search.proquest.com/docview/878136915?acc ountid $=14542$. 